\title{
Spectral Correlation in Ultrasonic Pulse Echo Signal Processing
}

\author{
Kevin D. Donohue, Member, IEEE John M. Bressler, Tomy Varghese, \\ Student Member, IEEE, and Nihat M. Bilgutay, Senior Member, IEEE
}

\begin{abstract}
This paper considers the effects of using spectral correlation in a maximum likelihood estimator (MLE) for backscattered energy corresponding to coherent reflectors embedded in media of microstructure scatterers. The spectral autocorrelation (SAC) function is analyzed for scatterer configurations based on the regularity of the interspacing distance between scatterers. It is shown that increased regularity gives rise to significant spectral correlation between different frequencies, while scatterers that are uniformly distributed throughout a resolution cell result in no significant correlation between spectral components. This implies that when a true uniform distribution for the effective scatterers exists, the power spectral density (PSD) is sufficient to characterize their echoes. However, as the microstructure scatterer distribution becomes more regular, SAC terms between different frequencies become more significant. Experimental results compare the performance of an adaptive MLE using the SAC and PSD characterizations of the grain echoes. The MLE results for 15 A-scans from stainless steel specimens with three different grain sizes indicate an average 6-dB signal-to-noise ratio (SNR) improvement in the coherent scatterer (flat-bottom hole) echo intensities for estimators using the SAC characterization, as opposed to estimators using the PSD characterization.
\end{abstract}

\section{INTRODUCTION}

$\mathbf{T}$ THIS paper presents a linear estimator, based on the maximum-likelihood estimator (MLE) derived by Donohue [1], for estimating the ultrasonic cross section (reflectivity) of isolated flaws and impurities within materials composed of regular microstructures using RF broadband ultrasonic Ascans. The estimator incorporates both the RF phase and magnitude distinctions between the spectra of the echoes from regular microstructure and from disruptions in the microstructure regularity. An adaptive implementation of this estimator is presented for estimating A-scan amplitudes corresponding to backscattered energy from coherent scatterers embedded in the microstructure of the material. This adaptive implementation processes A-scan segments corresponding to the duration of the illuminating pulse. The microstructure echoes are characterized from a neighborhood of sample points around the segment of interest. This segment is shifted over

Manuscript received June 3, 1992; revised December 10, 1992; accepted January 25,1993 . This work was supported in part by the National Science Foundation under Grant MIP-8920602. by the National Cancer Institute and the National Institutes of Health under Grant CA52823, and by the Biomedica Research Support Grant Program, Division of Research Resources, National Institute of Health under Grant BRSG S07 RR07114-23.

K. D. Donohue and T. Varghese are with the Department of Electrical Engineering, University of Kentucky, Lexington, KY 40506-0046.

J. M. Bressler and N. M. Bilgutay are with Department of Electrical and Computer Engineering, Drexel University, Philadelphia, PA 19104.

IEEE Log Number 9209519 the A-scan in a sliding window fashion and the estimator parameters are computed for each new segment position. The estimator parameters are updated at each new position in order to compensate for the nonstationary behavior of the backscattered energy received over the duration of the Ascan [2]. The nonstationary behavior results from frequency dependent absorption, scattering, and diffraction, which occurs as the pulse propagates through the material [3], [4].

Scattered energy from microstructures within materials limits the ability of ultrasonic pulse echo systems to detect small isolated flaws or changes in the microstructure properties. Several approaches have been taken to improve the detectability of target structures of interest in these situations. If the average size of the scatterers (which comprise the microstructure) is known a priori, then an optimal frequency range for the scanning transducer can be chosen to limit the sensitivity of the illuminating energy to these scatterers, while maintaining sensitivity to flaws and defects modeled by larger scatterers [3]. A related approach involves spectral filtering broadband pulse echo signals, which lends itself to adaptive filtering in cases where grain size is unknown or changing.

The utilization of greater detail from the microstructure echoes has been considered for nondestructive testing applications [5], [6], and medical applications [7], [8] (tissue characterization). For example, the work by Landini et al. [7] characterized the regular spacing of the microstructure scatterers with the spectral signature of the A-scan using gamma distribution parameters. It was demonstrated that both the average scatterer spacing and the regularity of that spacing could be characterized with gamma distribution parameters estimated from the periodicity and attenuation of the cepstral peaks. The work presented in this paper utilizes distribution properties of the microstructure scatterers in order to detect changes due to an embedded flaw or anomaly. Original contributions are made by introducing a spectral two-dimensional autocorrelation (SAC) function of the echo spectra for characterizing scatterer distribution properties, and using it in an estimator for detecting coherent scatterers embedded in the microstructure scatterers (i.e., small flaws in materials, or tumors in biological tissue). Experimental results indicate that significant improvements in the signal-to-noise ratio (SNR) occur when correlation coefficients of the Fourier spectrum are included in the characterization of the grain echoes.

Section II illustrates a relationship between the expected SAC values and regularity in the microstructure scatterers, where the effective interspacing distance of the microstructure 
scatterers is modeled by a gamma distribution. Section III discusses the application of the MLE to A-scans where regular microstructures may exist. Section IV presents experimental results demonstrating the significance of the SAC values on the MLE performance. Performance results for 15 different A-scans (stainless steel samples with 3 different grain sizes) indicate that when the SAC values were used, an average 6-dB improvement in SNR occurs over cases when only the power spectral density (PSD) values were used.

\section{SPECTRAL CORRELATON}

Common ultrasonic signal processing techniques employ deconvolution methods involving Wiener and matched filtering approaches [9], [10]. In these filters the PSD is typically used to characterize the effects of microstructure echoes and the system responses. The PSD indicates the correlation between the same frequency components of the return echo spectra. This is simply the expected energy for a random process as a function of frequency. Therefore, frequency shifts in the concentration of ultrasonic energy between the transmitted and received pulse (due to frequency dependent attenuation in propagation) are observed as changes in the PSD. This section considers cases where correlation exists between different spectral components of the return echo and its significance. It is shown that when scatterers are uniformly distributed, the expected values of the cross correlated frequency samples are insignificant. In this case, only the frequency samples correlated with themselves (equivalent to the PSD) dominate the SAC. When regularity exists for the scatterer positions, however, the correlation terms between different frequencies become significant. In the following discussion, neither the system response (illuminating pulse characteristics), nor the frequency dependent scattering is considered in order to isolate the effects of microstructure regularity.

Consider a distribution of scatterers relative to the axial A-scan distance denoted by:

$$
g(t)=\sum_{n=1}^{N} A_{n} \delta\left(t-T_{n}\right)
$$

where $t$ denotes the time axis (related to distance via the velocity of the pulse), $N$ is the total number of scatterers, $A_{n}$ denotes the strength or reflectivity of the $n$th scatterer, and $T_{n}$ represents the delay associated with the $n$th scatterer position. The Fourier transform of(1) is:

$$
G(f)=\sum_{n=1}^{N} a_{n} \exp \left(-j 2 \pi f \tau_{n}\right)
$$

where $a_{n}$ is the absolute value of $A_{n}$, and $\tau_{n}$ corresponds to $T_{n}+\pi$ for $A_{n}$ negative and to $T_{n}$ for $A_{n}$ positive. For a given configuration of scatterers, the SAC function is a complex surface obtained by correlating the values of $G(f)$ with its complex conjugate:

$$
\begin{gathered}
S\left(f_{1}, f_{2}\right)=G\left(f_{1}\right) G^{*}\left(f_{2}\right)=\sum_{n=1}^{N} \sum_{m=1}^{N} a_{n} a_{m} \\
\cdot \exp \left(-j 2 \pi\left(f_{1} \tau_{n}-f_{2} \tau_{m}\right)\right)
\end{gathered}
$$

where $f_{1}$ and $f_{2}$ are continuous variables forming the bifrequency plane. The SAC function in (3) is related to the spectral correlation density function [11], which is used to characterize multipath propagation and cyclostationary behavior in communication signals. The form of the SAC function used here is consistent with the covariance matrix used in the MLE presented in the next section. The effect of scatterer distribution properties on the SAC function is demonstrated via two cases.

For case one, consider the expected value of $S\left(f_{1}, f_{2}\right)$, where the random variables of (2) are taken to be the positive scattering strengths $\left(a_{i}\right)$ and associated phase $\left(\tau_{i}\right)$. Assume that the random variables associated with $a_{i}$ and $\tau_{i}$ are uncorrelated. If the phase related to the position of the scatterers within the resolution cell are uniformly distributed, the expected value for $S\left(f_{1}, f_{2}\right)$ can be written as:

$$
\begin{aligned}
E\left[S\left(f_{1}, f_{2}\right)\right]= & \frac{1}{\beta^{2}} \sum_{n=1}^{N} \sum_{\substack{m=1 \\
m \neq n}}^{N} E\left[a_{n} a_{m}\right] \\
& \int_{0}^{\beta} \int_{0}^{\beta} \exp \left(-j 2 \pi\left(f_{1} \tau_{n}-f_{2} \tau_{m}\right)\right) d \tau_{m} d \tau_{n} \\
& +\frac{1}{\beta} \sum_{n=1}^{N} E\left[a_{n}^{2}\right] \int_{0}^{\beta} \exp \left(-j 2 \pi \tau_{n}\left(f_{1}-f_{2}\right)\right) d \tau_{n}
\end{aligned}
$$

where $E[\cdot]$ is the expected value operator, and $\beta$ is the axial distance corresponding to the resolution cell (or signal window length). The two summation terms in (4) result from the correlation between two different scatterers (cross terms of (3)), and correlation of a scatterer with itself (quadratic terms of (3)). These integrate to obtain:

$$
\begin{aligned}
& E\left[S\left(f_{1}, f_{2}\right)\right]=\sum_{n=1}^{N} \sum_{\substack{m=1 \\
m \neq n}}^{N} E\left[a_{n} a_{m}\right] \exp \left(-j \pi \beta\left(f_{1}-f_{2}\right)\right) \\
& \operatorname{sinc}\left(\beta f_{1}\right) \operatorname{sinc}\left(\beta f_{2}\right)+\sum_{n=1}^{N} \mathrm{E}\left[a_{n}^{2}\right] \\
& \exp \left(-j \pi \beta\left(f_{1}-f_{2}\right)\right) \operatorname{sinc}\left(\beta\left(f_{1}-f_{2}\right)\right)
\end{aligned}
$$

where $\operatorname{sinc}(x)=\sin (\pi x) /(\pi x)$. Note that only the $\beta$ parameter (which relates to the window over which the scatterers are observed) affects the shape of the SAC function in (5). The expected values of the scatterer strengths in this equation mainly affect the overall magnitude of the SAC function and not the particular shape. The components of the SAC function that are off the $f_{1}=f_{2}$ axis are dominated by the $\operatorname{sinc}\left(\beta\left(f_{1}-f_{2}\right)\right)$ factor in the quadratic term, since the other sinc functions in the cross product terms will be significantly smaller (particularly for $f_{1}, f_{2}>(1 / \beta)$ ). The critical information derived from the $S A C$ function in this case lies mainly along the $f_{1}=f_{2}$ axis. In cases where significant frequency dependence of the scatterer strength exists (i.e., $E\left[a_{n} a_{m}\right]$ and $E\left[a_{n}^{2}\right]$ vary as functions of $f$ ) the PSD indicates the nature of the frequency dependent attenuation by magnitude variations as a function of frequency. Therefore, when the effective scattering centers are uniformly distributed throughout the observation cell, no significant 
correlation between different spectral components is expected, and the PSD is sufficient for characterizing the scatterers.

In the second case, consider regular spacing for the $N$ microstructure scatterers within the cell of interest. In this case the information concerning the average scatterer spacing and the regularity of that spacing affects the SAC function components for $f_{1} \neq f_{2}$. This is seen by performing a change of variables. Let:

$$
\Delta_{n}=\frac{\tau_{n}}{n}
$$

where $\tau_{n}$ is the effective time corresponding to the $n$th scattering center relative to the beginning of the cell. For a resolution cell containing $N$ effective scattering centers, $\tau_{n}$ corresponds to the scattering center where $n-1$ scattering centers precede the $n$th scattering center, and $N-n$ scattering centers follow. Therefore, the effective time delay of each of the scatterers can be expressed as $\tau_{n}=n \Delta_{n}$. Substitute this expression into (3) to result in:

$$
S\left(f_{1}, f_{2}\right)=\sum_{n=1}^{N} \sum_{m=1}^{N} a_{n} a_{m} \exp \left(-j 2 \pi\left(f_{1} n \Delta_{n}-f_{2} m \Delta_{m}\right)\right) \text {. }
$$

If scattering centers are equally spaced (i.e., $\Delta_{n}$ equals $\Delta$ for all $n$ ), (7) becomes:

$$
S\left(f_{1}, f_{2}\right)=\sum_{n=1}^{N} \sum_{m=1}^{N} a_{n} a_{m} \exp \left(-j 2 \pi \Delta\left(f_{1} n-f_{2} m\right)\right)
$$

If $S\left(f_{1}, f_{2}\right)$ is considered only for the PSD values $\left(f_{1}=f_{2}\right)$, then Eq. (8) reduces to:

$$
S(f, f)=\sum_{n=1}^{N} \sum_{m=1}^{N} a_{n} a_{m} \exp (-j 2 \pi \Delta f(n-m))
$$

Note that in (9), when $f$ is a multiple of $1 / \bar{\Delta}$, all terms in the summation are in phase, and a local maximum occurs. As a result of the periodic peak in the PSD, correlation between frequencies separated by $1 / \Delta$ is expected. Therefore, significant correlation values can be expected for spectral components when $f_{1}$ and $f_{2}$ are separated by multiples of $1 / \Delta$. In addition, these SAC values contain the expected phase differences between spectral components, such information is not available in PSD values. In the following discussion, the effect of increasing the variance associated with the average interspacing scatterer distance is examined.

Consider a gamma distribution for the $\Delta_{n}$ random variable given by:

$$
p(\Delta ; \nu, \bar{\Delta})=\frac{\left(\frac{\nu}{\Delta}\right)\left(\frac{\nu \Delta}{\bar{\Delta}}\right)^{\nu-1}}{(\nu-1) !} \exp \left(\frac{-\nu \Delta}{\bar{\Delta}}\right)
$$

where $\bar{\Delta}$ is the average interdistance scatterer spacing ( $\bar{\Delta}=$ $\mathrm{E}[\Delta])$, and $\nu$ is the order of the gamma distribution, which is related to the variance of the interdistance spacing ( $\mathrm{E}[(\Delta-$ $\left.\bar{\Delta})^{2}\right]=\bar{\Delta}^{2} / \nu$ ). The gamma distribution is used here because it is a flexible model for representing scatterer regularity. It can be used to model a wide variety of scatterer distributions, from nearly uniform $(\nu<10)$ to almost deterministic $(\nu>1000)$. The expected value of the SAC function, when $\Delta$ is an
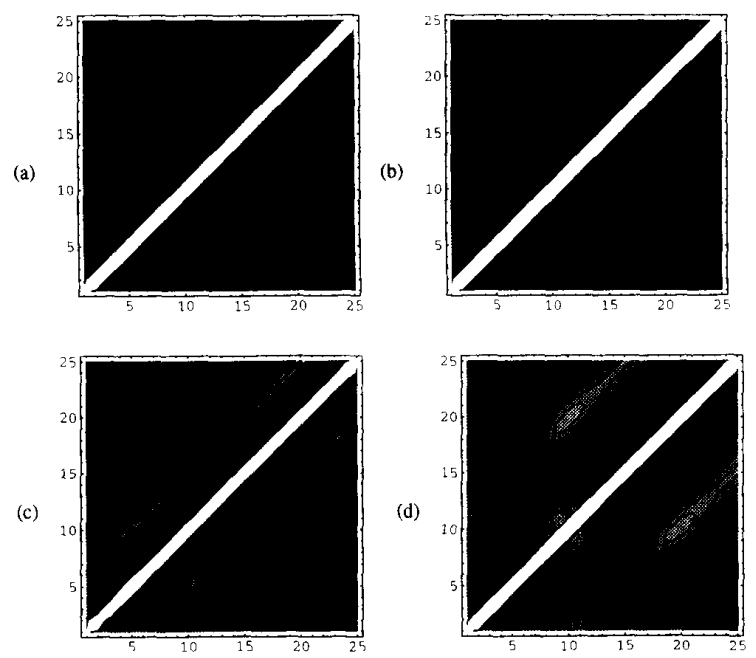

Fig. 1. Contour plots for expected SAC magnitude functions in the bi-frequency plane $\left(f_{1}, f_{2}\right.$ axes in megahertz) for randomly placed scatterers with gamma distributed interdistance spacing $\bar{\Delta}$ and order $\nu$. (a) $\bar{\Delta}=0.2 \mu \mathrm{s}$, $\nu=10$. (b) $\bar{\Delta}=0.1 \mu \mathrm{s}, \nu=10$. (c) $\bar{\Delta}=0.2 \mu \mathrm{s}, \nu=100$. (d) $\bar{\Delta}=0.1 \mu \mathrm{s}$ $\nu=100$.

independent gamma distributed random variable, is given by:

$$
\begin{aligned}
& E\left[S\left(f_{1}, f_{2}\right)\right] \\
& =\sum_{\substack{n=1 \\
m \neq n}}^{N} \sum_{\substack{m=1 \\
N}}^{N} E\left[a_{n} a_{m}\right] \alpha\left(n f_{1}, \nu, \bar{\Delta}\right) \\
& \quad \alpha\left(m f_{2}, \nu, \bar{\Delta}\right) \exp \left(-j 2 \pi\left(\phi\left(\bar{\Delta}, \nu, n f_{1}\right)-\phi\left(\bar{\Delta}, \nu, m f_{2}\right)\right)\right) \\
& \quad+\sum_{n=1}^{N} E\left[a_{n}^{2}\right] \alpha\left(n\left(f_{1}-f_{2}\right), \nu, \bar{\Delta}\right) \\
& \quad \cdot \exp \left(-j 2 \pi \phi\left(\bar{\Delta}, \nu, n\left(f_{1}-f_{2}\right)\right)\right)
\end{aligned}
$$

where:

$$
\alpha(f, \nu, \bar{\Delta})=\left(1+\left(\frac{2 \pi \bar{\Delta} f}{\nu}\right)^{2}\right)^{\frac{-\nu}{2}}
$$

and

$$
\phi(\bar{\Delta}, \nu, f)=\nu \tan ^{-1}\left(2 \pi f \frac{\bar{\Delta}}{\nu}\right) .
$$

Fig. 1 presents contour density plots of the SAC magnitude for 4 different cases of (11). For these plots the scatterer strength values are assumed wide sense stationary with an independent Rayleigh distribution. This implies that $E\left[a_{n} a_{m}\right]$ (for $m \neq n$ ) equals $(\pi / 4) E\left[a_{n}^{2}\right]$ for all $m$ and $n$. In addition, only $1 \mu \mathrm{s} / \bar{\Delta}$ scatterers (truncate to integer value) are included in the summation, with the SAC function evaluated at 25 frequencies from 1 to $25 \mathrm{MHz}$. Fig. 1 (a) and (c) has the same average interspacing scatterer distance of $\bar{\Delta}=0.2 \mu \mathrm{s}$, with different orders $\nu$. Likewise, Fig. 1 (b) and (d) has the average interspacing scatterer distance of $\bar{\Delta}=0.1 \mu \mathrm{s}$, with different orders $\nu$.

When $\nu$ equals 10 , very little regularity exists in the scatterer spacing. As a result Fig. 1 (a) and (b) resembles the case for uniformly distributed scatterers (4). The peak of this function 

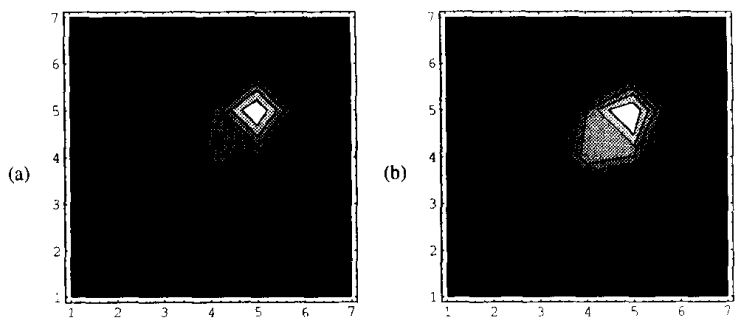

Fig. 2. Contour plots for expected SAC magnitude functions estimated from stainless steel A-scan data. The bi-frequency plane is labeled in terms of the DFT index ( 1 corresponds to dc, and 7 corresponds to $9.5 \mathrm{MHz}$ ): (a) Sample with average grain size $86 \mu \mathrm{m}$. (b) Sample with average grain size $160 \mu \mathrm{m}$.

exists as a line along the $f_{1}=f_{2}$ diagonal. Even at this low order, the nature of the energy concentration around the diagonal is sensitive to the underlying scatterer spacing. When the order is increased to 100 , as in Fig. 1 (b) and (c), local maxima in the off-diagonal components due to the regular spacing are observed. Note that the local maxima components are located $1 / \Delta \mathrm{Hz}$ away from the center diagonal in the horizontal and vertical directions. Local maxima along the PSD values also occurs at multiples of $f_{1}=f_{2}=5 \mathrm{MHz}$ in Fig. 1 (c), and $10 \mathrm{MHz}$ in Fig. 1 (d). The PSD peaks are not detectable at higher multiples of $1 / \bar{\Delta} \mathrm{Hz}$, even when observing direct plots of the PSD [12]. The SAC, however, indicates correlation between these spectral regions.

The phase plots are difficult to present and graphically interpret due to the discontinuities from the $2 \pi$ modularity, and therefore, are not presented. However, it was observed from examining various phase plots that the phase changes more rapidly over the anti-diagonal of the SAC for the scattering structures with greater interspacing distance. This result is expected from the scaling relationship between $\left(f_{1}-f_{2}\right)$ factor and $\bar{\Delta}$ in function $\phi(\cdot)(13)$ as used in the quadratic term of (11). In general, the phase in the off-diagonal terms indicates an average phase difference between corresponding spectral regions.

In this section a relationship between the scatterer spacing distribution and the SAC function was demonstrated. Other effects were not considered, such as non-Rayleigh distributions of the scattering strengths, frequency dependence of the scatterer strength, effects of the pulse echo system response, and nongamma distributions for the scattering spacing. If quantitative estimates of distribution parameters are required, such effects must be considered. Fig. 2 presents sample SAC functions estimated from twenty 64 point A-scan segments from stainless steel echoes. The limited bandwidth of the pulse echo system results in a limited frequency window over which the expected SAC function is observed. The resultant SAC is a point by point multiplication of the system response SAC function and the expected SAC from the scatterers. Note that the SAC function for stainless steel resembles the low-order gamma function (i.e., no local minimum in the off-diagonal region), which is expected for echoes from randomly oriented grain boundaries. In other materials, such as biological tissue [12], a more regular interdistance scatterer spacing can result in discernible local minima in the off-diagonal terms.
The SAC function can be directly applied in an adaptive filter to characterize echoes from spatial regions known to contain only microstructure. Neighboring regions can be tested based on this characterization, to determine if the echoes significantly deviate from the previously characterized $\mathrm{mi}$ crostructure echoes. In the next section, a direct application of the SAC is presented for detecting isolated disruptions in the microstructure by an embedded coherent scatterer. This is applied adaptively to reduce the need for precise knowledge of the material ultrasonic properties.

\section{THE MLE FOR A-SCAN AMPLITUDES FROM A COHERENT SCATTERER}

This section considers extending the application of the MLE derived by Donohue [1] to include the case when regularity exists in the microstructure scatterers. The MLE was based on sampled A-scan segments (corresponding to an echo from a resolution cell of interest) modeled in terms of its DFT components (frequency samples). It was assumed that each DFT component for an echo received from a resolution cell had a complex Gaussian distribution. The target scatterer was modeled as an embedded coherent scatterer within the resolution cell. The MLE for the target ultrasonic cross section, $\alpha$, was given by:

$$
\alpha_{m l}(R ; \tau, \Sigma, S)=\frac{R^{*} \Sigma^{-1} T S+S^{*} T^{*} \Sigma^{-1} R}{2 S^{*} T^{*} \Sigma^{-1} T S}
$$

where $R$ is a vector of complex elements representing the significant DFT components of the received echo (those corresponding to the bandwidth of the illuminating pulse), $\tau$ denotes the relative position of the coherent scatterer within the resolution cell ( $\tau$ equal to zero corresponds to the center), $\Sigma$ is the covariance matrix for the jointly distributed DFT components, $S$ is a vector denoting the DFT components of the illuminating energy within the resolution cell, and $T$ is a diagonal matrix representing the phase shift on each component of $S$ due to a nonzero $\tau$. For the implementation described later in this paper, processing is done with respect to the center of the cell such that $\tau$ is zero, and $T$ is the identity matrix. Therefore, the critical components for implementing this estimator are $\Sigma$ and $S$.

The covariance matrix $\Sigma$ is effectively a sampled version of the SAC introduced in the previous section. Therefore, the diagonal elements of $\Sigma$ are samples of the microstructure echo PSD. The MLE in [1] characterized DFT samples of the RF microstructure echoes within a given resolution cell by a stationary zero-mean Gaussian distribution, which resulted in $\Sigma$ being a diagonal matrix. In this paper, (14) is considered when the microstructure can be characterized in off-diagonal terms for $\Sigma$. If the DFT coefficients can be characterized by a joint zero-mean Gaussian distribution for a nonstationary process, (14) is the MLE estimate of the coherent scatterer cross section. In cases where the distribution of the DFT coefficients deviate from a joint Gaussian distribution (i.e., due to a small number of scatterers per resolution cell), the optimal processor will typically require coefficients for higher order (nonlinear) terms of the received signal. Such deviations are not considered in this paper. 
In order to implement (14) on A-scan data, $S$ and $\Sigma$ must be determined. Since the $\Sigma$ matrix represents the expected scattered energy from the unresolvable scatterers, it can be estimated from the A-scan data under the assumptions that negligible attenuation and similar microstructure regularity exists over a neighborhood of resolution cells, and that no target scatterer exists within this neighborhood (not including the resolution cell of interest). The $S$ vector in the MLE corresponds to DFT coefficients of the illuminating pulse at a given resolution cell. This parameter is determined from the characteristics of the transducer that generated the pulse, such as the center frequency, bandwidth, and pulseshape. As distortion and attenuation occur during propagation, $S$ must be adjusted by accounting for the previously scattered energy. This updating process, however, cannot be performed exactly since the backscattered energy is measured over a finite spatial window. The choice of $S$ can be simplified, if a weak scattering assumption is applied. The error that results from this simplification is discussed in [1]. For the A-scans presented in this paper, this approximation is not expected to generate significant error, since relatively little attenuation of the backscattered energy occurs over the duration of the A-scan (see Fig. 3). Therefore, for the implementation described in this paper, the elements of $S$ remain the same for all resolution cells.

It also was shown in [1] that the processor of (14) was algorithmically equivalent to the matched filter (correlation receiver), when only diagonal elements of $\Sigma$ were used. Therefore, the inclusion of the off-diagonal terms can be interpreted as an extension of the matched filter to include correlation between frequency components. The matched filter interpretation is helpful when considering the meaning and effects of the $S$ vector. In [1] it was assumed that the coherent scatterer is large enough such that no distortion (frequency dependent scattering) occurs for the echo of interest over the spectrum of the pulse echo. The $\Sigma^{-1}$ matrix characterizes the distortion due to the microstructure, and whitens echo spectra similar characteristics, while the $S$ vector characterizes the undistorted echo, and enhances echoes of similar spectral characteristics. Therefore, the more the echo resembles the spectrum determined by the $S$ vector, and the less it resembles the echoes characterized by $\Sigma$, the larger the MLE output.

The elements of $S$ effectively window the received DFT coefficients. The significant elements of $S$ (corresponding to the approximate 3-dB bandwidth of the transducer) define the effective number of DFT components required for the formula given in (14). Those elements of $S$ that are relatively small and outside the bandwidth of the transducer are set to zero. Since the magnitude of $S$ is not adjusted to reflect the true energy incident upon the resolution cell, the computed $\alpha_{m l}$ is not a true ultrasonic cross section of the coherent scatterer. Instead, these estimates represent relative amplitudes corresponding to backscattered energy from the coherent scatterer.

Since the estimator in (14) corresponds to scatterers located at the center sample in the resolution cell of interest (i.e., $\tau=0$ and $T$ is the identity matrix), this suggests processing the A-scan in a sliding window fashion, such that every Ascan sample can be at the center of the processing window.

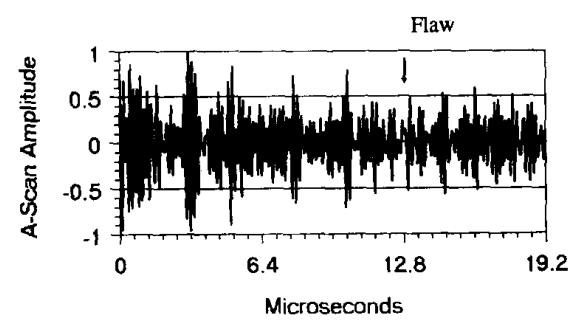

(a)

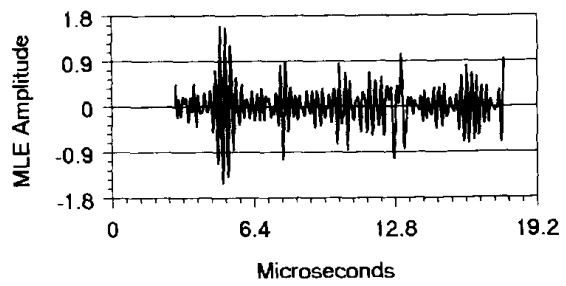

(b)

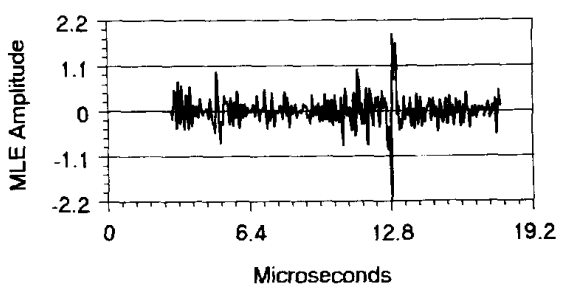

(c)

Fig. 3. Comparison of A-scan amplitude estimations. (a) Original A-scan with flat-bottom hole corresponding to the indicated flaw position. (b) Processed A-scan for MLE using only diagonal elements of covariance matrix. ( c) Processed A-scan for MLE using all elements of covariance matrix.

Let the samples for the resolution cell under consideration be denoted by vector:

$$
\boldsymbol{r}(n, L)=[r(n-L / 2), \cdots r(n), \cdots, r(n+L / 2)]^{T}
$$

where $r(n)$ is the $n$th time sample of the RF A-scan, $n$ is the center of the sample relative to the resolution cell, and $L+1$ is the number of samples in the resolution cell $(L+1$ should be chosen to correspond to the number of samples in a pulsewidth), and superscript $T$ denotes the transpose operation. Therefore, to check for a target scatterer at all possible samples in the A-scan, the window described by $r(n, L)$ can be applied at all A-scan points, and the MLE performed for each increment of $n$.

In order to perform the MLE on the segment described in (15), sample points must be chosen for estimating $\Sigma$. The examples presented in this paper use samples on both sides of the resolution cell of interest (a noncausal implementation). Let a data segment, used to estimate $\Sigma$, be denoted by the vector:

$$
\boldsymbol{r}_{i}(n, L)=[r(n+i L / 2-L / 2), \cdots, r(n+i L / 2+L / 2)]^{T}
$$

where $n$ denotes the center of the segment in which the estimation is being performed, and $i$ is the index for neigh- 
boring segments (a negative $i$ represents segments prior to the segment of interest). Note that each increment of $i$ results in a $50 \%$ overlap between adjacent segments, which is done to improve efficiency of the SAC estimates from the given data. Each segment used to estimate $\Sigma$ is multiplied by a Hamming window, and a DFT is taken of the segment. The $\Sigma$ matrix is then estimated from the resulting DFT vectors by:

$$
\Sigma(n)=\sum_{i=2}^{W} R_{-i}(n, L) R_{-i}^{*}(n, L)+R_{i}(n, L) R_{i}^{*}(n, L)
$$

where $R_{i}(n, L)$ is the DFT vector of $r_{i}(n, L)$, and $2(W-1)$ is the total number of segments used to estimate $\Sigma$. Note that $i$ begins with 2 in order to prevent the segments used in estimating $\Sigma$ from overlapping with the segment of interest. In general, to create a full rank $k$ by $k$ covariance matrix, at least $k$ independent $\mathrm{A}$-scan segments should be used to create the $R_{i}(n, L)$ vector needed in (17). In addition, only DFT components with significant energy should be used from the $R_{i}(n, L)$ vector to create $\Sigma$. These are components typically within the 3- to 6-dB range of the transducer.

\section{COMPARISON OF EXPERIMENTAL RESULTS}

This section presents performance results for two cases of the MLE applied to experimental A-scans from stainless steel cylinders of various grain sizes. In the first case $\Sigma$ is restricted to a diagonal matrix (PSD values), and in the second case the off-diagonal terms are also included (SAC values). Sample covariance matrices were estimated from grain-only A-scans and significant terms were observed in the off-diagonal elements. The values in the first off-diagonal terms ranged in magnitude from 25 to $70 \%$ of the diagonal elements. The results in this section demonstrate that these terms are significant in discriminating between resolution cells containing only microstructures and those that include a coherent scatterer.

The A-scans used in this comparison were obtained from three 2-in diameter stainless steel rods that were heat treated to obtain various grain sizes. A fiaw was simulated in each specimen by drilling a flat-bottom hole of $4.22-\mathrm{mm}$ diameter. The samples were placed in a water bath and scanned with a 1/2-in KB-Aerotech Alpha transducer with a center frequency of $5 \mathrm{MHz}$ and a Gaussian-shaped spectrum. The received echoes were digitized at a sampling rate of $100 \mathrm{MHz}$, and each measurement was then averaged 200 times in a LeCroy 9400 digital oscilloscope to reduce time varying noise. Average grain sizes for the three samples were 86,106 , and $160 \mu \mathrm{m}$. These values were determined from micrographs using a linear intercept method [13].

The time-domain Gaussian-shaped pulse, which approximated the illuminating pulse for each resolution cell, corresponded to a window duration of 56 samples. The cut-off points defining the time-domain pulse duration were the points where the Gaussian function was $97 \%$ down from the peak value [1]. The 3-dB transducer bandwidth was approximately $2.5 \mathrm{MHz}$. The resolution cell samples were increased to 64 samples so that an FFT could be applied. In addition, only 4 DFT components corresponded to the bandwidth of the segmented A-scan, and 5 segments, one on each side of the segment of interest, were used in computing $\Sigma$ (i.e., $W=6$ in (17)). Thus the effective dimension of $\Sigma$ used in the MLE computations was 4 by 4 , and the $S$ parameter was approximated by a Gaussian window with zero (linear) phase. Problems associated with the limited frequency resolution are discussed at the end of the section.

An example of an unprocessed A-scan for the $160-\mu \mathrm{m}$ average grain size is shown in Fig. 3 (a). The results of processing this A-scan with the MLE using the diagonal elements of $\Sigma$ is shown in Fig. 3 (b), while the results for the case where all elements of $\Sigma$ were used is presented in Fig. 3 (c). Note for this particular case the MLE only uses diagonal elements which failed to estimate an A-scan amplitude for the flaw that was high enough to be distinguished from the grain noise. However, the MLE using the full covariance matrix succeeded in discriminating the flaw from the grain echoes.

Further results are presented in terms of the ratio between peak flaw intensity to peak grain echo intensity within a given A-scan. The intensity is obtained from an RF A-scan by sliding a window of a length equal to the resolution cell (i.e., 64 samples) over each sample in the A-scan. Every element in the window is squared and then averaged. The resulting intensity corresponds to the sample position at the center of the window. The highest peak associated with the flaw echo resolution cell is divided by the highest peak associated with the grain echoes. This ratio is expressed in decibels and referred to as the peak SNR. Note that a peak SNR value greater $0 \mathrm{~dB}$ implies that the A-scan intensity corresponding to the flaw is greater than the largest intensity corresponding to the grain. In this case a threshold exists which can detect the flaw with no false alarms. For the A-scans shown in Fig. 3, only Fig. 3 (c) would result in a peak SNR greater than 0 . The flaw in the intensity images corresponding the A-scans in Fig. 3 (a) and (b) cannot be detected by a threshold test without false alarms.

Fig. 4 compares the peak SNR results for both cases of the MLE. Five A-scans were obtained from each stainless steel specimen by moving the transducer relative to the flat-bottom hole, such that the hole remained within the beam field. Each set of three bars on the graph indicate the peak SNR for the original A-scan and the two cases for the processed A-scan, which are the MLE using only the diagonal elements of its covariance matrix, and the MLE using all elements in the covariance matrix.

Fig. 4 (a) presents the results for five different A-scans obtained from the $160 \mu \mathrm{m}$ average grain size specimen. Note that in every case the MLE with the full covariance matrix significantly enhanced the SNR (on the order of a $10 \mathrm{~dB}$ increase), while the MLE with the diagonal covariance matrix failed to enhance the SNR above $0 \mathrm{~dB}$ in three of the cases ( $\mathrm{A}$ scan numbers 1,4 , and 5). The performance for the other two cases (A-scan numbers 2 and 3 ) is consistent with examples presented in [1]. In these cases the flaw was more centered in the interrogating beamwidth.

On average, the MLE with the full covariance matrix performed better than the MLE with the diagonal covariance matrix by about $6 \mathrm{~dB}$. Fig. 4 (b) and (c) present SNR results of 106- and $86-\mu \mathrm{m}$ average grain size specimens. Similar 
(a)

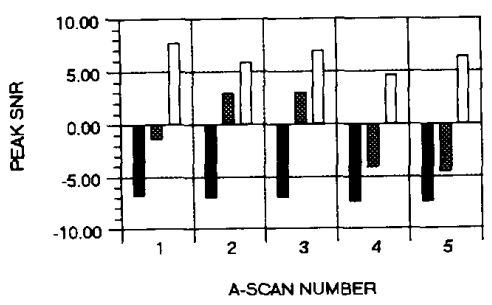

(b)

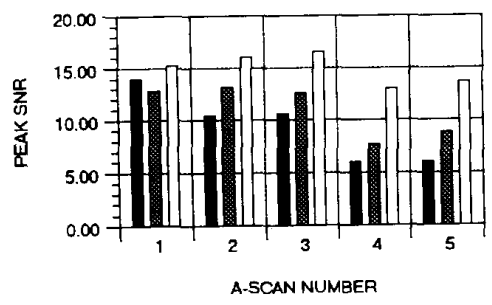

(c)

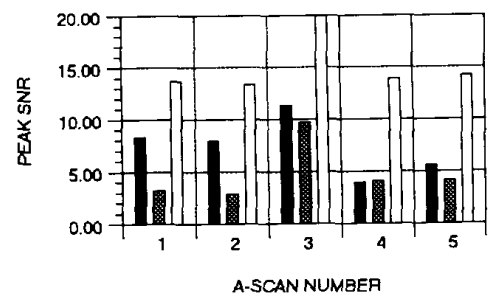

Original A-Scan

圆 MLE with Diagonal Covariance Matrix

$\square$ MLE with Full Covariance Matrix

Fig. 4. Comparison of SNR for the two cases of the MLE. (a) Five A-scans from $160-\mu \mathrm{m}$ average grain size stainless steel specimen. (b) Five A-scans from $106-\mu \mathrm{m}$ average grain size stainless steel specimen. (c) Five A-scans from $86-\mu \mathrm{m}$ average grain size stainless steel specimen.

enhancement results are observed; however, in these cases enhancement is not as critical as in Fig. 4 (a), since the original SNR is greater than $0 \mathrm{~dB}$ to begin with. The purpose of these results is to demonstrate the consistency of the estimator. Further experimental results and details on implementing the MLE are presented by Bressler in [14].

The inconsistency of the MLE that utilized only PSD values can be attributed to the limited resolution in the frequency domain. Discrimination based on the PSD characterization relies on differences between the grain and target echo spectra. The grain echoes tend to have a higher frequency content, and as a result simple bandpass filtering can be applied [2], [4]. In these examples, however, estimating the spectra with the DFT algorithms on the 64 point segments limits the resolution in the frequency domain, and therefore, reduces the power to discriminate between the target and grain echo spectra. In cases where a significant SNR existed to begin with, some losses in SNR resulted from the diagonal covariance matrix MLE due to distortion from the low resolution estimates of the PSD. On the other hand, the MLE that utilized the off-diagonal

terms of the covariance matrix performed well in spite of the limited resolution in the frequency domain. Results presented by Bressler [14] indicate improved performances for the MLE using the PSD characterization when the processing cell size was increased from 64 to 128 samples per cell (note the spatial resolution is reduced in this case).

\section{CONCLUSIONS}

The experimental results indicate that using SAC values enhances the detectability of isolated flaws embedded in grain noise, over simply using the PSD information. This is particularly true when limited differences exist between the power spectra for the flaw and the grain, and some form of regularity exists for the grain structures. While a relationship was demonstrated between the spacing of the scattering sites and the off-diagonal terms of the covariance matrix, the ability of the covariance matrix to characterize distribution properties of the scatterers is not limited to this form of regularity. Other effects such as distribution of scattering strength, frequency dependent scattering, and dispersion can also affect the offdiagonal terms of the covariance matrix. The actual cause for the off-diagonal terms in the MLE covariance matrices that improved the performance for the data used was not identified in this paper. It is believed that consistencies in the grain configurations, observed over short segments, result in loworder regularity for the effective scattering center positions. The SAC function did not characterize the scatterer spacings corresponding to the actual grain diameters, since a $160-\mu \mathrm{m}$ spacing in a material with a $5.8-\mathrm{km} / \mathrm{s}$ velocity corresponds to a $1 \longdiv { \Delta }$ equal to $36 \mathrm{MHz}$. This would not be detected over the 4- to $5-\mathrm{MHz}$ bandwidth used in the experimental results presented in this paper. It is most likely that the effective scattering centers correspond to clusters of grain scatterers (or isolated grain boundaries at particular orientations) related to the grain distribution and the illuminating energy. These notions are currently being examined via computer simulation studies.

Experimental results indicate that the application of the SAC characterization in the MLE can improve the detectability of small flaws or defects within a resolution cell. Since no quantitative relationship was presented for the $\mathrm{SAC}$ and grain structure, this application is limited to adaptive implementations where the covariance matrix can be estimated for a given resolution cell of interest. The stability of the inverse computation for $\Sigma$ was not a problem in processing the experimental data. If for a given application, however, illconditioned matrices occur (usually seen by large sporadic spikes in the processed A-scan), stability can be improved by either including more independent data segments in the $\Sigma$ estimation, or by eliminating DFT coefficients on either end of the transducer spectrum, where they may be significantly smaller in magnitude than the coefficients in the rest of the spectrum.

\section{REFERENCES}

[1] K. D. Donohue, "Maximum-likelihood estimation of A-scan amplitudes for coherent targets in media of unresolvable scatterers," IEEE Trans. Ultrason., Ferroelect., Freq. Contr., vol. 39, May 1992 
[2] J. Xin, K. D. Donohue, and N. M. Bilgutay, "Filter design for ultrasonic flaw echo detection in large-grained materials," in Review of Progress in Quantitative NDE, vol. 10A. New York: Plenum, 1991, pp. 725-731.

[3] J. Krautkramer, and H. Krautkramer, Ultrasonic Testing of Materials, 4th ed. New York: Springer-Verlag, 1990, p. 113.

[4] P. M. Shankar, U. Bencharit, N. M. Bilgutay, and J. Saniie, "Grain noise suppression through bandpass filtering," Mat. Evaluation, vol. 46, no. 8 , pp. $1100-1104$, July 1988 .

[5] F. S. Cohen, "Modeling of ultrasound speckle with application in flaw detection in metals," IEEE Trans. Signal Proc., vol. 40, pp. 624-632, Mar. 1992.

[6] N. M. Bilgutay, X. Li, and J. Saniie, "Spectral analysis of randomly distributed scatterers for ultrasonic grain size estimation," Ultrason., vol. 27, pp. 19-25, Jan. 1989

[7] L. Landini, and L. Verrazzani, "Spectral characterization of tissues microstructure by ultrasounds: A stochastic approach," IEEE Trans. Ultrason. Ferroelect. Freq. Contr., vol. 37, pp. 448-455, Sept. 1990

[8] M. Insana, R. F. Wagner, D. G. Brown, and T. Hall, "Describing smallscale structure in random media using pulse-echo ultrasound," $J$. Acoust. Soc. Amer., vol. 87, no. 1, pp. 179-192, Jan. 1990.

[9] M. Fatemi and A. C. Kak, "Ultrasound B-scan imaging: Theory of image formation and technique for restoration," Ultrason. Imaging, vol. 4, no. 2, pp. $1-47,1980$.

[10] G. S. Kino, Acoustic Waves Devices, Imaging \& Analog Signal Processing. Englewood Cliffs, NJ: Prentice-Hall, 1987, pp. 488-498.

[11] W. A. Gardner, "Exploitation of spectral redundancy in cyclostationary signals," IEEE Signal Proc. Mag., vol. 8, no. 2, pp. 14-36, Apr. 1991.

[12] K. D. Donohue and T. Varghese, "Spectral cross correlation for tissue characterization," in Proc. 1992 IEEE Ultrasonic. Symp., Tucson, AZ, Oct. 1992.

[13] N. M. Bilgutay and J. Saniie, " The effect of grain size on flaw visibility enhancement using split-spectrum processing," Mat. Evaluation, vol. 42, pp. 808-814, May 1984.

[14] J. M. Bressler, "Maximum likelihood estimation of ultrasonic reflectivity in nonstationary noise," M.S. thesis, Drexel Univ., Philadelphia, PA, May 1991.

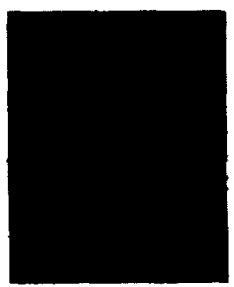

Kevin D. Donohue (S'84-M'87) was born in Oak Park, IL, on April 19, 1958. He received the B.A. degree in mathematics from Northeastern Illinois University, Chicago, IL, in 1981, and the B.S., M.S., and Ph.D. degrees from Illinois Institute of Technology, Chicago, IL, in 1984, 1985, and 1987. respectively.

In 1985, he was an engineer for Zenith Electronics, in Glenview, $\Pi$, developing ultrasonic detection circuits. From 1989 to 1991 he was a Visiting Assistant Professor at Drexel University doing research in radar and ultrasonic signal detection. Since 1991 he has been an Assistant Professor in the Electrical Engineering Department at the University of Kentucky. His current research interests include detection and estimation theory, statistical pattern recognition, and image processing for applications in nondestructive testing of materials and medical imaging.

Dr. Donohue is a member of SPIE and Sigma Xi.

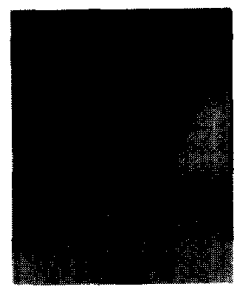

John M. Bressler was born in Philadelphia, PA, on June 7, 1965. He received the B.S. and M.S. degrees in electrical engineering from Drexel University, in Philadelphia, PA, in 1988 and 1991, respectively.

His research interests include the application of a maximum likelihood estimator of ultrasonic reflectivity to A-scan signals collected by insonifying steel blocks of various average grain sizes. $\mathrm{He}$ is currently a Software Engineer for FAAC, Incorporated, in Huntingdon Valley, PA.

Mr. Bressler is a member of Eta Kappa Nu.

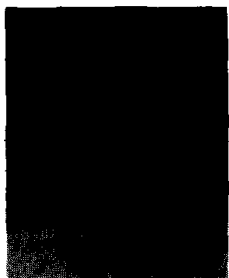

Tomy Varghese ( $S^{\prime} 92$ ) was born in Bangalore, India, on January 26,1967 . He received the B.E. degree in instrumentation technology from the University of Mysore, India, in 1988, and the M.S degree in electrical engineering from the University of Kentucky, Lexington, KY, in 1992. He is currently working toward the Ph.D. degree from the University of Kentucky.

From 1988 to 1990 has was employed as a Engineer in Wipro Information Technology Ltd, India His current research interests include detection and estimation theory, statistical pattern recognition, signal and image processing applications in medical imaging, and analog/digital hardware design.

Mr. Varghese is a member of the Eta Kappa Nu.

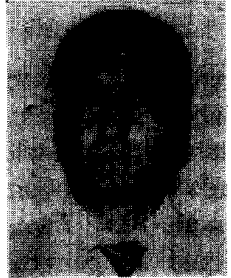

Nihat M. Bilgutay (M'81-SM'87) was born in Ankara, Turkey on March 31, 1952. He received the B.S. degree in electrical engineering from Bradley University, Peoria, $\Pi$, in 1973, and the M.S. and Ph.D. degrees in electrical engineering from Purdue University, West Lafayette, IN, in 1975 and 1981 respectively.

He is currently a Professor in the Electrical and Computer Engineering Department and Associate Dean for Graduate Programs and Research at the College of Engineering at Drexel University. His research activities and interests include ultrasonic imaging, nondestructive testing and material characterization, digital signal processing, and communication theory.

Dr. Bilgutay is a member of Tau Beta Pi, Eta Kappa Nu, Sigma Xi, ASEE and ASNT. 